\title{
Adaptation of Pseudomonas sp. AKS2 in biofilm on low-density polyethylene surface: an effective strategy for efficient survival and polymer degradation
}

\author{
Prosun Tribedi, Anirban Das Gupta and Alok K Sil
}

\begin{abstract}
Background: Pseudomonas sp. AKS2 can efficiently degrade low-density polyethylene (LDPE). It has been shown that this degradation of LDPE by AKS2 is correlated to its ability to form biofilm on the polymer surface. However, the underlying mechanism of this biofilm-mediated degradation remains unclear. Since bioremediation potential of an organism is related to its adaptability in a given environment, we hypothesized that AKS2 cells undergo successful adaptation in biofilm on LDPE, which leads to higher level of LDPE degradation. To verify this, the current study investigated a number of parameters of AKS2 cells in biofilm that are known to be involved in adaptation process.

Results: Successful adaptation always develops a viable microbial population. So we examined the viability of AKS2 cells in biofilm. We observed the presence of viable population in the biofilm. To gain an insight, the growth of AKS2 cells in biofilm on LDPE at different time points was examined. Results showed a better reproductive competence and more colonization for AKS2 biofilm cells than planktonic cells, indicating the increased fitness of AKS2 biofilm cells than their planktonic counterpart. Towards understanding fitness, we determined the hydrolytic activity, different carbon source utilization potentials, functional diversity and homogeneity of AKS2 biofilm cells. Results showed increased hydrolytic activity (approximately 31\%), higher metabolic potential, higher functional diversity (approximately 27\%) and homogeneity for biofilm-harvested cells than planktonic cells. We also examined cellular surface hydrophobicity, which is important for cellular attachment to LDPE surface. Consistent with the above results, the cell surface hydrophobicity of biofilm-harvested AKS2 cells was found to be higher (approximately 26\%) compared to that of their planktonic counterpart. All these results demonstrated the occurrence of physiological as well as structural adaptations of AKS2 cells in biofilm on LDPE surface that resulted in better attachment, better utilization of polymer and better growth of AKS2 cells, leading to the development of a stable colony on LDPE surface.

Conclusions: The present study shows that AKS2 cells in biofilm on LDPE surface undergo successful adaptation that leads to enhanced LDPE degradation, and thus, it helps us to understand the underlying mechanism of biofilm-mediated polymer degradation process by AKS2 cells.
\end{abstract}

Keywords: Polyethylene-based plastic material; Bioremediation; Pseudomonas; Biofilm; Adaptation

* Correspondence: alokksil7@gmail.com

Department of Microbiology, University of Calcutta, 35 B.C. Road, Kolkata 700019, India 


\section{Background}

In the modern era, plastic-based materials have a variety of domestic and industrial applications. However, the widespread use of this non-biodegradable material poses a major threat to the environment. An example of a widely used non-biodegradable polymer is polyethylene. Although there are reports of microbial degradation of polyethylene, the rate is very slow $[1,2]$. Moreover, this microbial degradation requires the pre-oxidation of polyethylene, either by physical or chemical treatment [1-4]. Previously, we reported that Pseudomonas sp. AKS2 can degrade $5 \% \pm 1 \%$ of low-density polyethylene (LDPE) in just 45 days, without any prior oxidation of polyethylene [5]. This report also documented that AKS2 developed biofilm on polyethylene surface efficiently, and there was a linear correlation between this biofilm formation and the ability to degrade polyethylene [5]. However, the underlying mechanism of this biofilm-mediated LDPE degradation by AKS2 remains unclear.

Biofilm represents a complex association of microorganisms in a given habitat [6], and its formation is a bacterial survival response to hostile environment [7]. Microorganisms are known to be capable of altering their structural and physiological activities through biofilm formation as this allows survival under varied environmental conditions. Such alteration in activities for better survival in a given habitat is known as adaptability.

In general, adaptation is a biological process by which an organism becomes more competent to live in a given habitat [8]. Following adaptation, microorganisms exhibit different physiological and structural activities compared to their non-adapted counterparts [9]. Thus, adaptation provides a kind of biological insurance for an organism to encounter varying environments in a given ecological niche. The adaptive traits may be structural, behavioural or physiological. Structural adaptation includes variations in shape and size of the organism. The alteration of membrane fluidity, by both psychrophilic and thermophilic bacteria, is an example of structural adaptation. This type of modulation in membrane fluidity serves as a protection against harsh temperatures. While behavioural adaptations are composed of inherited behaviour chains, physiological adaptations allow the organism to perform special functions for the adjustment of cellular growth and development, regulation of temperature, etc. Bacterial secretion of exo-polysaccharides for their attachment to a solid surface is an example of physiological adaptation.

Existing literature documents that bioremediation potential of an organism is related to its adaptability in a given environment [10]. Adapted Rhodococcus erythropolis DCL14 cells were shown to degrade alkanes and alcohols at higher rate compared to their non-adapted counterparts [11]. Thus, we hypothesized that successful adaptation of AKS2 population in biofilm on LDPE surface resulted in its sustained retention at this site, which resulted in enhanced polymer degradation.

To verify this hypothesis, we investigated the various parameters of AKS2 structure and physiology such as fitness, metabolic potential, functional diversity and homogeneity that are relevant to the adaptation. We observed increased fitness, higher levels of functional diversity and homogeneity, and increased cell surface hydrophobicity for biofilm adapted cells compared to the planktonic cells, indicating the physiological and structural adaptations of AKS2 cells in biofilm. Thus, these results demonstrate that successful adaptation of AKS2 cells in biofilm on LDPE surface resulted in enhanced degradation of LDPE.

\section{Methods}

Bacterial strain and culture condition

Pseudomonas sp. AKS2 was previously isolated from Kolkata municipal solid waste dumping ground soil (Kolkata, India) [12]. It is a potential degrader of polyethylene succinate (PES) [12] and LDPE [5]. This isolate was grown in $100 \mathrm{ml}$ of sterile basal media containing $300 \mathrm{mg}$ of sterile LDPE films at $30^{\circ} \mathrm{C}$ for different time periods as per the requirement of the experiments. Basal media were prepared as described previously [5]. Commercially available LDPE was used in all the experiments. LDPE films were made additive free by washing with $70 \%$ ethanol. The surface area of each polyethylene film used was $5 \times 4 \mathrm{~cm}$.

\section{Dual staining for the assessment of AKS2 viability in biofilm}

To determine the viability of AKS2 cells in biofilm on polyethylene surface after the incubation, LDPE films were removed from the conditioned medium and the adhered bacterial population, if any, were stained with $4 \mu \mathrm{g} \mathrm{ml}^{-1}$ acridine orange for $15 \mathrm{~min}$ [5]. Thereafter, LDPE films were washed with sterile Milli-Q water (Millipore Corporation, Billerica, MA, USA) and further treated with $4 \mu \mathrm{g} \mathrm{ml}^{-1}$ ethidium bromide for another $15 \mathrm{~min}$. LDPE films were again washed with sterile Milli-Q water. Thereafter, dried films were observed under a fluorescence microscope (Olympus IX 71, Olympus Corporation, Tokyo, Japan).

\section{Measurement of AKS2 fitness}

Microbial fitness represents the ease of reproduction of an organism in a given environment. In order to examine the fitness of AKS2 biofilm cells, we compared the colonization and reproduction potential of biofilmharvested cells with that of planktonic AKS2 cells. For this experiment, AKS2 cells were grown for 30 days in 
media containing LDPE films as sole C-source. After the incubation, cells that adhered to LDPE films were extracted. These cells represent biofilm-harvested cells. To compare the colonization and reproduction potential, biofilm-harvested and planktonic AKS2 cells were inoculated separately in equal numbers (approximately $10^{4}$ cells) into $100 \mathrm{ml}$ of basal media containing $300 \mathrm{mg}$ of LDPE film as sole C-source and incubated at $30^{\circ} \mathrm{C}$ for different lengths of time. After incubation for 5 and 10 days, LDPE films were taken out from the growth media and examined under a fluorescence microscope after staining with acridine orange as described in the previous section. All experiments were performed in triplicate.

\section{Fluorescein diacetate hydrolysis assay}

To examine the hydrolytic activity of bacterial cells either harvested from biofilm or planktonic condition, fluorescein diacetate (FDA) hydrolysis assay was performed [13]. Briefly, equal numbers of cells (approximately $10^{6}$ cells) taken from the respective origin were separately added to $1.5 \mathrm{ml}$ of $60 \mathrm{mM}$ sodium phosphate buffer, $\mathrm{pH}$ 7.6. FDA solution was added to it to attain a final concentration of $10 \mu \mathrm{g} \mathrm{ml}^{-1}$. The flask was then shaken at $30^{\circ} \mathrm{C}$ for $30 \mathrm{~min}$. These samples were then centrifuged at 6,000 rpm for $5 \mathrm{~min}$, and the absorbance of the supernatant was measured by a spectrophotometer (V-630, Jasco, Tokyo, Japan) at $494 \mathrm{~nm}$. Samples without FDA served as a control.

\section{Evaluation of bacterial cell surface hydrophobicity}

Cell surface hydrophobicity of biofilm-harvested cells and planktonic cells was measured by bacterial adhesion to hydrocarbon (BATH) assay [14]. For this purpose, equal numbers of biofilm-harvested and planktonic AKS2 cells (approximately $10^{6}$ cells) were added separately to several tubes containing increasing volumes (ranging from 0 to $0.2 \mathrm{ml}$ ) of $\mathrm{n}$-hexadecane. Tubes were then shaken for $10 \mathrm{~min}$ and allowed to stand for $15 \mathrm{~min}$ to complete the phase separation. The $\mathrm{OD}_{400}$ of the aqueous suspensions was measured. Cell surface hydrophobicity was calculated using the following formula:

$$
\begin{aligned}
& \text { Cell surface hydrophobicity (in \%) } \\
& \quad=100 \times(\text { Initial OD-Final OD) } / \text { Initial OD }
\end{aligned}
$$

\section{Physiological profiles of AKS2 population}

The patterns of potential carbon source utilization by biofilm-harvested AKS2 cells and their planktonic counterpart were assessed using BiOLOG-ECO plates (Biolog, Bremen, Germany) containing triplicates of 31 different environmentally relevant carbon sources [15]. To perform the experiment, $150 \mu \mathrm{l}$ of either biofilm- harvested cells or planktonic cells containing approximately $10^{3} \mathrm{CFU}$ was separately added into each well of the BiOLOG-ECO plates. The plates were incubated at $30^{\circ} \mathrm{C}$ for $72 \mathrm{~h}$, and the absorbance of each well was recorded at $590 \mathrm{~nm}$. Microbial metabolic activity in each microplate, expressed as average well colour development (AWCD), was determined as follows: $\mathrm{AWCD}=\Sigma \mathrm{Absorbance}_{\mathrm{i}} / 31$, where Absorbance $_{\mathrm{i}}$ is the absorbance at $590 \mathrm{~nm}$ from each well. The Shannon diversity index $(H)$, an indicator of functional diversity, was calculated using the following equation: $H=-\Sigma \mathrm{pi} \ln \mathrm{pi}$, where pi is the ratio of the activity on each substrate (Absorbance $)_{\mathrm{i}}$ ) to the sum of activities of all substrates ( $\sum$ Absorbance $_{\mathrm{i}}$ ) and assuming absorbance at $590 \mathrm{~nm}$ of 0.25 as threshold for positive response [16]. The corresponding Lorenz curve that provides a graphical depiction of the information contained in the Shannon diversity index was plotted. Thereafter, this curve is used to derive the Gini coefficient $(G)$, which is a measure of functional inequality, using the formula:

$$
G=1-2 \int_{0}^{1} L \mathrm{~d} F
$$

where $L$ is the Lorenz curve and $F$ is the standardized cumulative distribution of the standardized population.

\section{Cluster analysis}

For cluster analysis, data from the richness tests using BiOLOG-ECO plates were collected from either biofilmharvested cells or planktonic cells of AKS2. The similarity matrix was generated by Euclidean distances, which were used to build a dendrogram with the unweighted pair group mean averages (UPGMA) algorithm wherein the linkage was single. Cluster analysis was performed by using the software Minitab 16.

\section{Statistical analysis}

Experimental results were subjected to statistical analysis of one-way analysis of variance (ANOVA) in order to evaluate statistically significant differences among samples. Mean values were compared at different levels of significance using the software Minitab 16. All experiments were performed in triplicate.

\section{Results}

AKS2 cells exhibit increased fitness for their growth on LDPE surface

In a habitat, an efficient adaptation of an organism should lead to the development of a viable population. Therefore, to verify the hypothesis that AKS2 cells undergo adaptation in biofilm, we examined the viability of AKS2 cells in biofilm on LDPE surface by performing dual staining with ethidium bromide and acridine 
orange. Acridine orange stains both the dead and viable cells, whereas ethidium bromide selectively stains the dead cells as it cannot pass through the intact membrane of living cells. Consistent with our previously published report [5], we observed AKS2 biofilm formation from day 30 onwards and a considerable weight loss of LDPE film (5\%) after incubation with AKS2 for 45 days (Additional file 1: Figure S1 and Additional file 2: Figure S2). Dual staining of LDPE film obtained after 30 days of incubation with AKS2 showed a large number of green cells (stained with acridine orange) in contrast to a few red cells (stained with ethidium bromide), indicating that majority of AKS2 cells present in biofilm were live (Figure 1). This result demonstrated the development of viable AKS2 population on polyethylene surface signifying an adaptation of AKS2 cells in biofilm on LDPE surface.

To better understand the adaptation, we examined the fitness of AKS2 cells in biofilm. Fitness is the ability of an organism to survive as well as reproduce in a given environment, and thus, successful adaptation is represented by better fitness. To examine the fitness of AKS2 cells in biofilm, we compared their colonization and reproduction potential on LDPE surface with that of planktonic AKS2 cells (see 'Methods' for details). For this purpose, biofilm-harvested and planktonic AKS2 cells were inoculated separately in equal numbers (approximately $10^{4}$ cells) into basal media containing LDPE film as sole C-source and incubated for different lengths of time. The LDPE films were then examined under a fluorescence microscope after staining with acridine orange to monitor the extent of AKS2 attachment to LDPE. The result showed greater adherence to the LDPE film by biofilm-harvested cells than planktonic cells (Figure 2A). We also compared the reproduction efficiency between biofilm-harvested cells and planktonic cells of AKS2 and observed an approximately 2.5-fold increase in cell number for biofilm-harvested cells from day 5 to day 10 (Figure 2B). For planktonic cells, the corresponding fold increase over the same period of time was only approximately 1.4-fold (Figure 2B). These results indicate that biofilm-harvested cells have higher reproduction ability than their planktonic counterparts. Thus, there is a significant increase in the fitness of biofilm-harvested AKS2 cells compared to that of their planktonic counterpart.

\section{Biofilm-harvested AKS2 cells exhibit increased functional diversity and metabolic activity}

In an ecological niche, metabolic functional diversity influences productivity of an organism by increasing their ability to utilize a greater variety of nutrients [17]. Thus, it is possible that in the biofilm, AKS2 cells increase metabolic functional diversity, which in turn enhances their LDPE degradation capability. To verify this, we compared the functional diversity of biofilmharvested and planktonic AKS2 cells by determining the Shannon diversity index from the utilization spectrum of 31 different eco-sensitive carbon sources in BiOLOG-ECO plates $[18,19]$. We observed that the Shannon diversity index is significantly higher (approximately 27\%) for biofilm-harvested cells than for planktonic cells (Figure 3A). To further validate the result, we compared metabolic potentials of biofilm-harvested cells and planktonic cells. Metabolic potential of an organism was examined by measuring the AWCD of BiOLOG-ECO plate [18]. As expected, the biofilmharvested cells of AKS2 showed higher level of AWCD compared to the planktonic cells (Figure 3B). Thus, the results demonstrated increased functional diversity and metabolic potential of biofilm-harvested AKS2 cells.

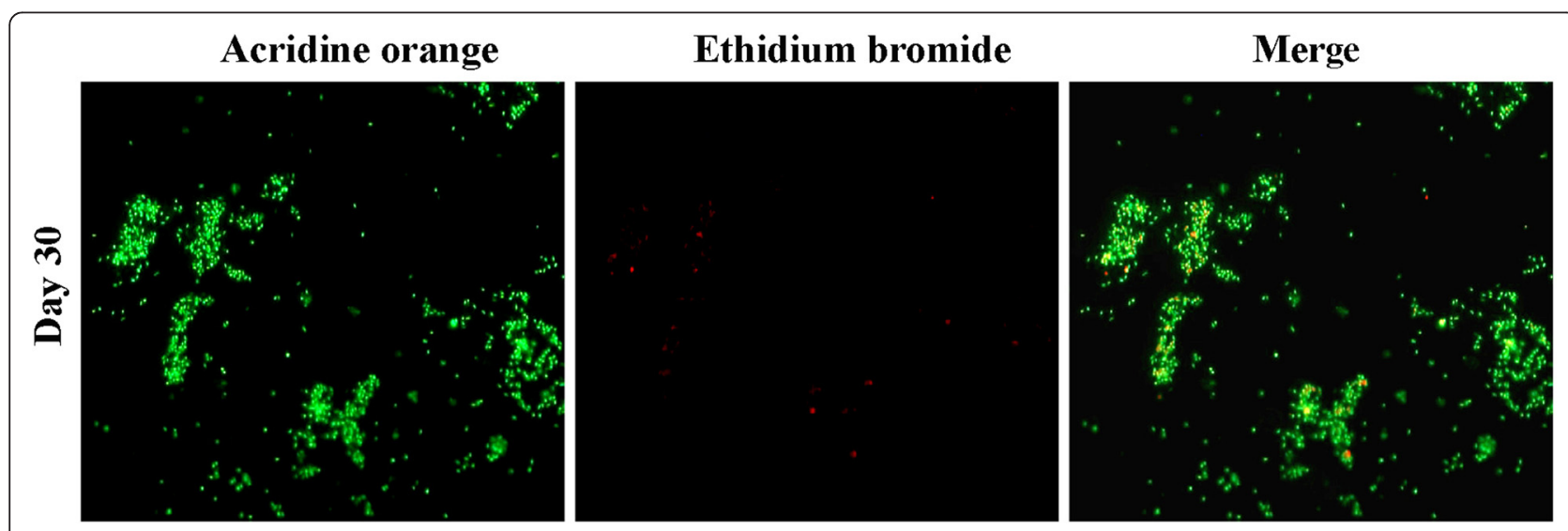

Figure 1 AKS2 develops viable microbial population in biofilm on polyethylene surface. LDPE films recovered from the conditioned media after 30 days of incubation were stained with acridine orange and ethidium bromide and observed under a fluorescence microscope. The figure is a representative of images obtained from 20 different fields and from three independent experiments. 


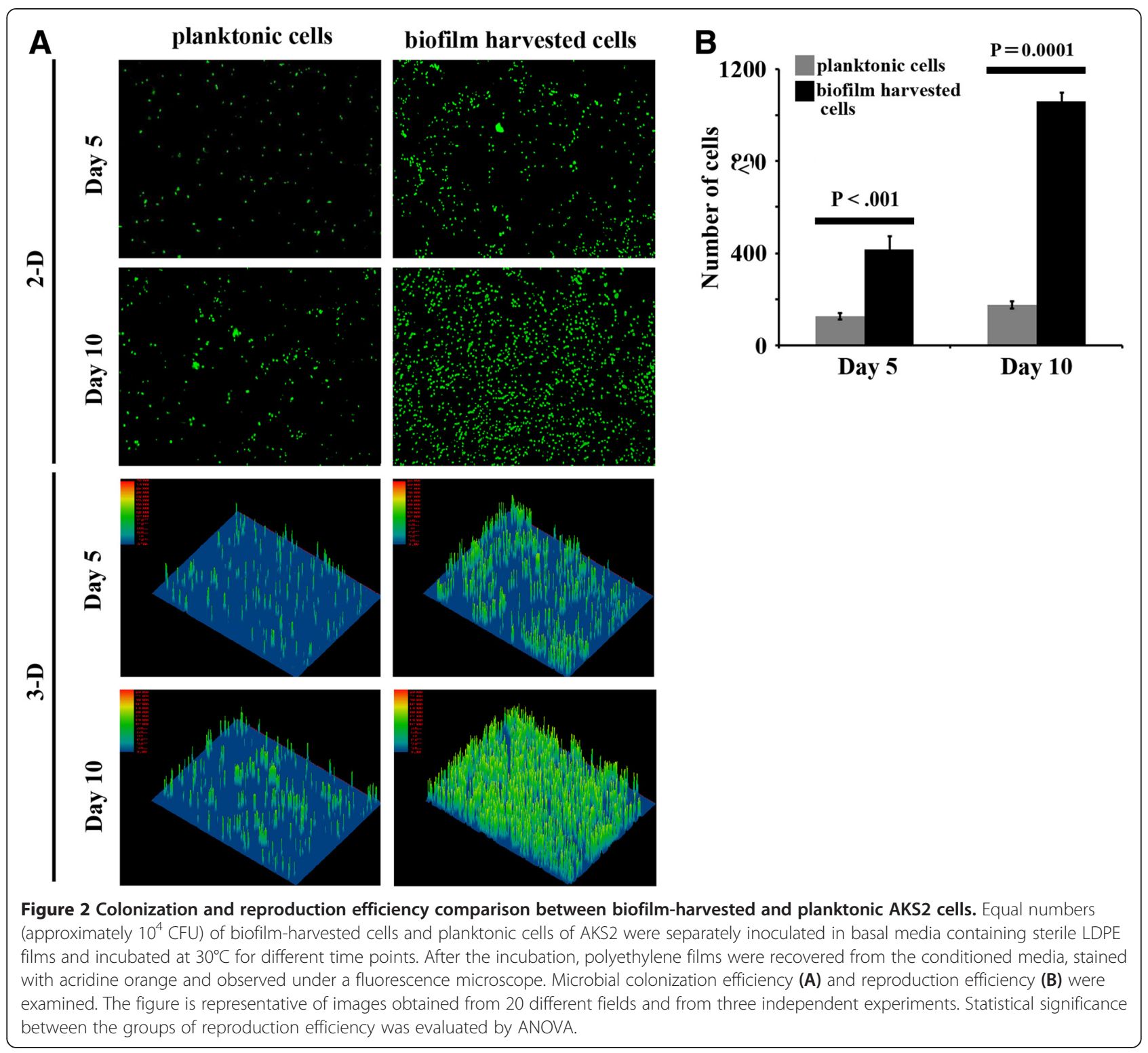

Viable microbial cells produce a large array of hydrolytic enzymes, which can cleave FDA to produce fluorescein that can be detected spectrophotometrically, and this assay is widely used to measure cell metabolic activity $[18,20]$. FDA is sensitive to esterase and lipase activity as it contains an ester linkage. Therefore, to verify the metabolic activity, we compared the FDA hydrolysis activity of biofilm-harvested AKS2 cells with the corresponding activity of AKS2 planktonic cells. The result showed higher level (approximately 31\%) of FDA hydrolysis activity in the extract obtained from biofilm-harvested AKS2 cells than in the extract from the planktonic cells (Figure 4). This result indicates that biofilm-harvested AKS2 cells harbour higher level of hydrolytic enzymes, which ensures the enhanced metabolic activity for biofilm cells than for planktonic cells.
AKS2 cells exhibited increased functional homogeneity in biofilm

Functional homogeneity or evenness of a microbial population contributes towards the development of a stable colony, and therefore, we determined the functional homogeneity of biofilm-harvested cells. To examine it, we plotted the Lorenz curves deduced from different carbon source utilization patterns by AKS2 under different conditions. Lorenz curve is the graphical representation of the degree of inequality in a population. The Lorenz curve for biofilm-harvested cells was found to be closer to the line of equality compared to their non-adapted planktonic counterpart (Figure 5A). The closer the curve to the line of equality, the higher the evenness will be, i.e., the system is more homogenously distributed. Thus, this result indicates higher 

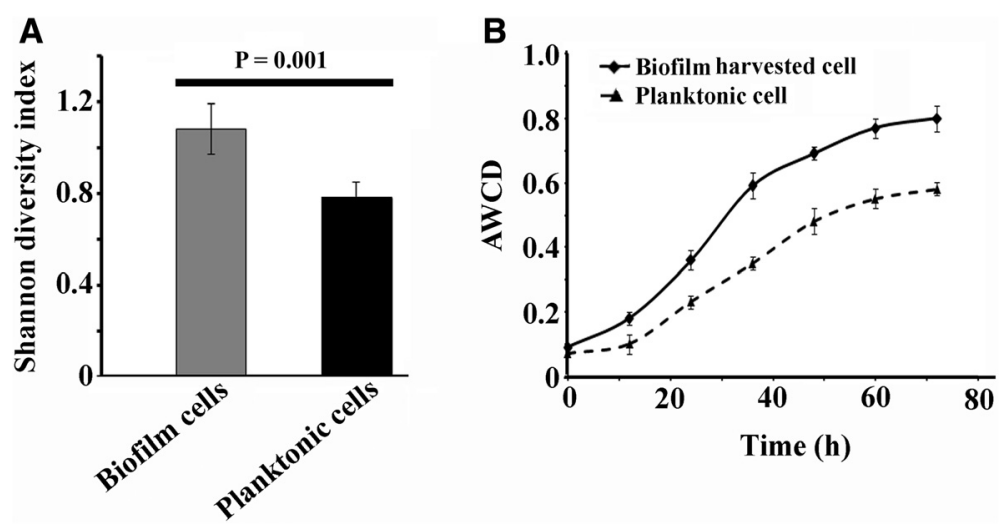

Figure 3 Functional diversity and metabolic activity profile of biofilm-harvested and planktonic AKS2 cells. Equal numbers (approximately $10^{3}$ (FU) of either biofilm-harvested or planktonic cells of AKS2 were separately added to each well of BiOLOG-ECO plate and incubated for 3 days at $30^{\circ} \mathrm{C}$. Absorbance at $590 \mathrm{~nm}$ of each well was recorded at different time points. Shannon diversity index (A) and average well colour development (B) were derived from well colour absorbance and plotted. Three replicates have been used for each experiment, and the result represents the average of these three replicates. Error bars indicate standard deviation $( \pm \mathrm{SD})$. Statistical significance between the groups was evaluated by ANOVA.

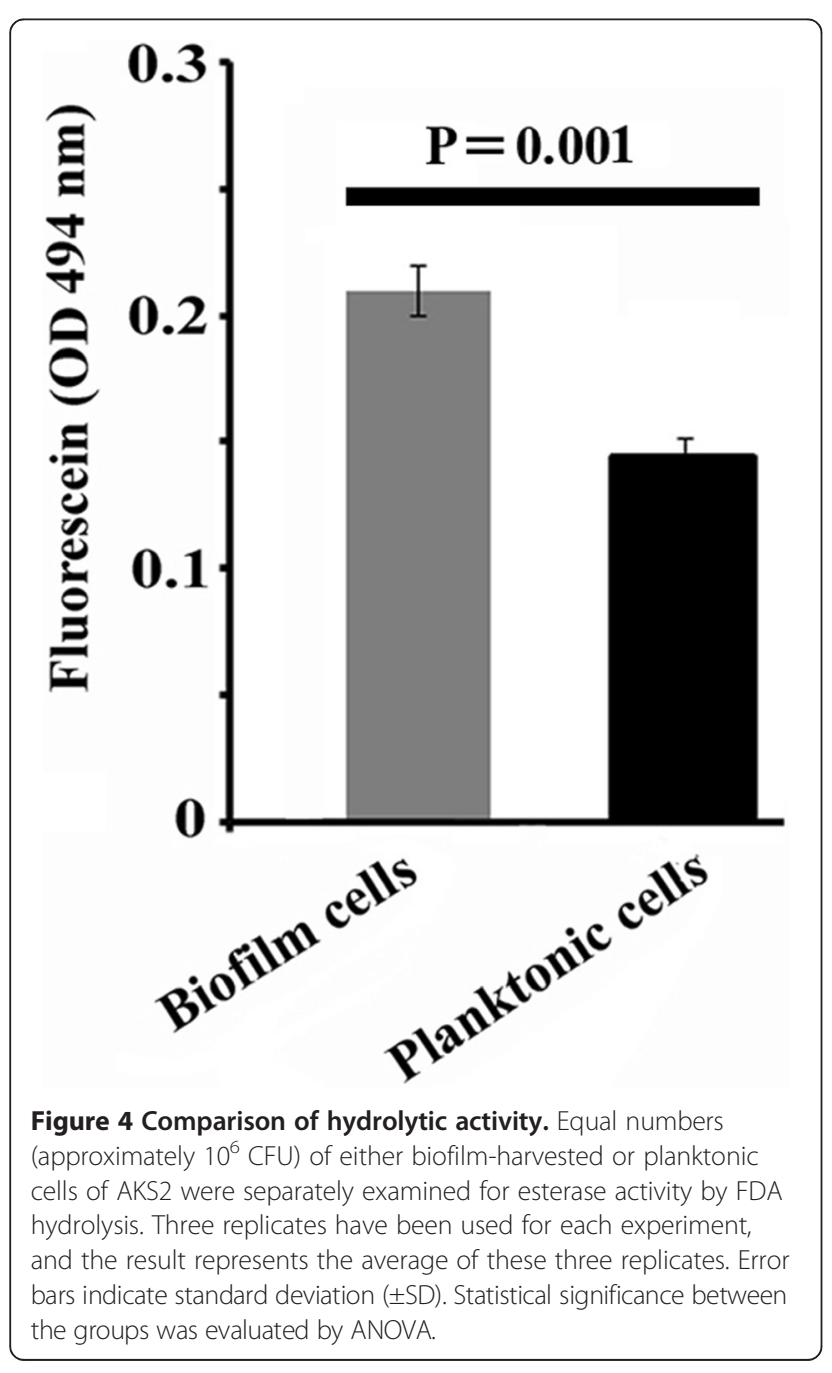

level of functional homogeneity for biofilm-harvested cells than planktonic cells. The subsequent analysis of Gini coefficient, a widely used inequality coefficient, showed a reduction in the coefficient value for biofilm-harvested cells compared to the planktonic cells (Figure 5A). Since evenness of a system varies inversely with the Gini coefficient, this result again indicates that AKS2 cells in biofilm have a higher degree of evenness with respect to their ability to utilize different C-sources than their planktonic counterpart. To lend support to this observation, we have also performed metabolic cluster analysis. AKS2 cells harvested from biofilm showed distinct and different patterns of metabolic cluster from their planktonic form (Figure 5B). Unlike planktonic cells, biofilm-harvested cells showed a large cluster (represented by a red colour) where most of the carbon sources were utilized with maximum similarity (Figure 5B). This result further confirms greater functional homogeneity or evenness for AKS2 cells in biofilm than for planktonic cells. Again, the increased evenness or functional homogeneity of AKS2 cells in biofilm contributes to better degradation of the polymer. Taken together, the increased levels of functional diversity, metabolic activity specially the hydrolytic activity and functional homogeneity indicate the occurrence of physiological adaptation of AKS2 cells on LDPE surface.

\section{Biofilm-harvested AKS2 cells exhibit higher level of cell surface hydrophobicity}

The cell surface hydrophobicity significantly contributes to cellular attachment to polymer surface, and thus, it plays an important role towards biofilm formation and polymer degradation as it brings the substrate (LDPE polymer) in close proximity to the enzyme $[5,21,22]$. 


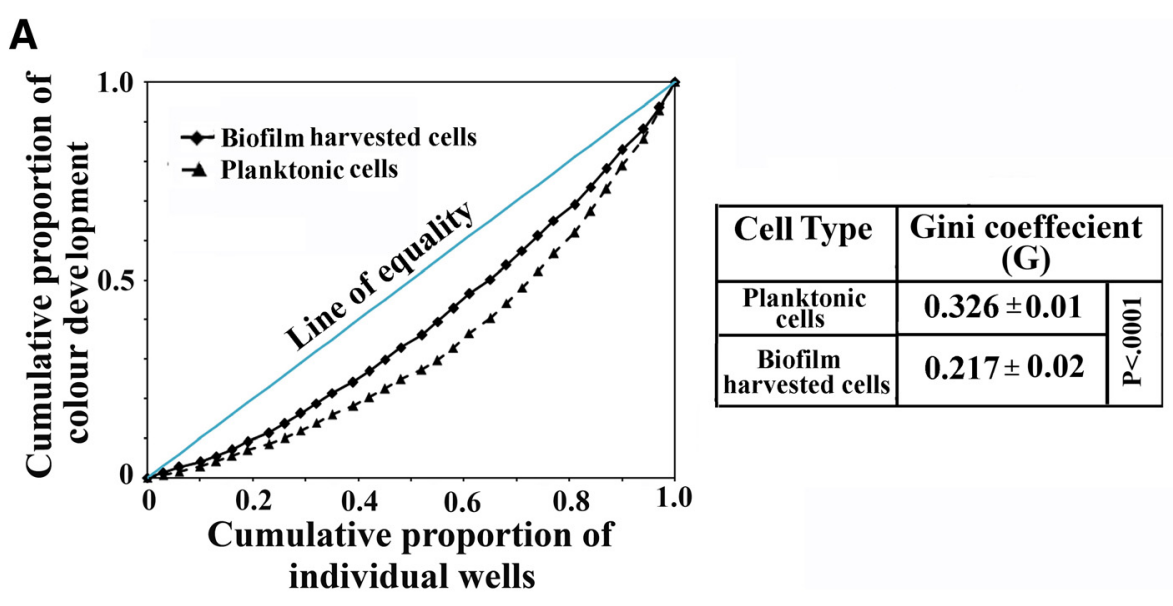

\section{B}
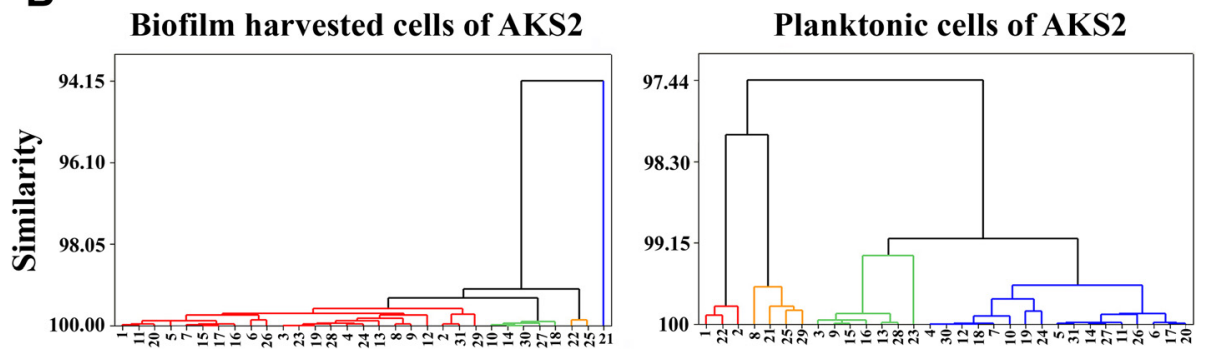

Figure 5 Biofilm-harvested AKS2 cells exhibit higher evenness. Equal numbers (approximately $10^{3}$ CFU) of either biofilm-harvested or planktonic cells of AKS2 were separately added to each well of BiOLOG-ECO plate and incubated for 3 days at $30^{\circ} \mathrm{C}$. Absorbance at $590 \mathrm{~nm}$ of each well was recorded at different time points. (A) Lorenz curves and Gini coefficient. (B) UPGMA cluster analysis. Statistical significance between the groups of Gini coefficient was evaluated by ANOVA.

Therefore, we examined the cell surface hydrophobicity of biofilm-harvested AKS2 cells and compared the same with planktonic cells. The result showed significantly higher (approximately 26\%) level of surface hydrophobicity in biofilm-harvested cells than planktonic AKS2 cells (Figure 6). It indicates the occurrence of structural adaptation of AKS2 cells in biofilm on LDPE surface.

\section{Polyethylene surface modulates the adaptation of AKS2}

It is possible that LDPE surface may contribute towards the observed adaptation of AKS2 cells on its surface as abiotic components are known to play an important role in the stability of an ecosystem [23]. Towards understanding the role of LDPE in this adaptation, we compared functional diversity and evenness of AKS2 biofilm cells on this polymer with those of AKS2 biofilm cells formed on another polymer, polyethylene succinate (PES), as these two components have direct correlation with adaptation. We observed a significant difference in functional diversity and evenness between biofilm cells taken from each polymer (Figure 7A,B). Since we started this experiment with an equal number of AKS2 cells for both the polymers, the different levels of functional diversity can only be attributed to the difference in the polymeric surface. Thus, this result clearly suggests a possible role of each polymer in the adaptation process of AKS2 in a given habitat.

\section{Discussion}

The present study investigated structural and physiological properties of AKS2 cells during LDPE degradation. It has been reported that adapted marine microorganisms can survive in extremely unfavourable environmental conditions containing high concentrations of pollutants and toxic substances like heavy metals, hydrocarbons, xenobiotics and other recalcitrant compounds by forming biofilm [10]. Similarly, LDPE degradation by AKS2 was also found to be increased concomitantly with the increased biofilm formation [5]. Though biofilm was shown to enhance bioremediation, the high cell density inside a biofilm is known to cause a stressful environment $[24,25]$. The ability of an organism to adapt to the different microenvironments in biofilm is an important survival strategy against this environmental stress [26,27]. In addition, a previous report has documented that under stressful conditions, microorganisms undergo phenotypic diversification to enhance their adaptive potential [28]. Towards understanding the 


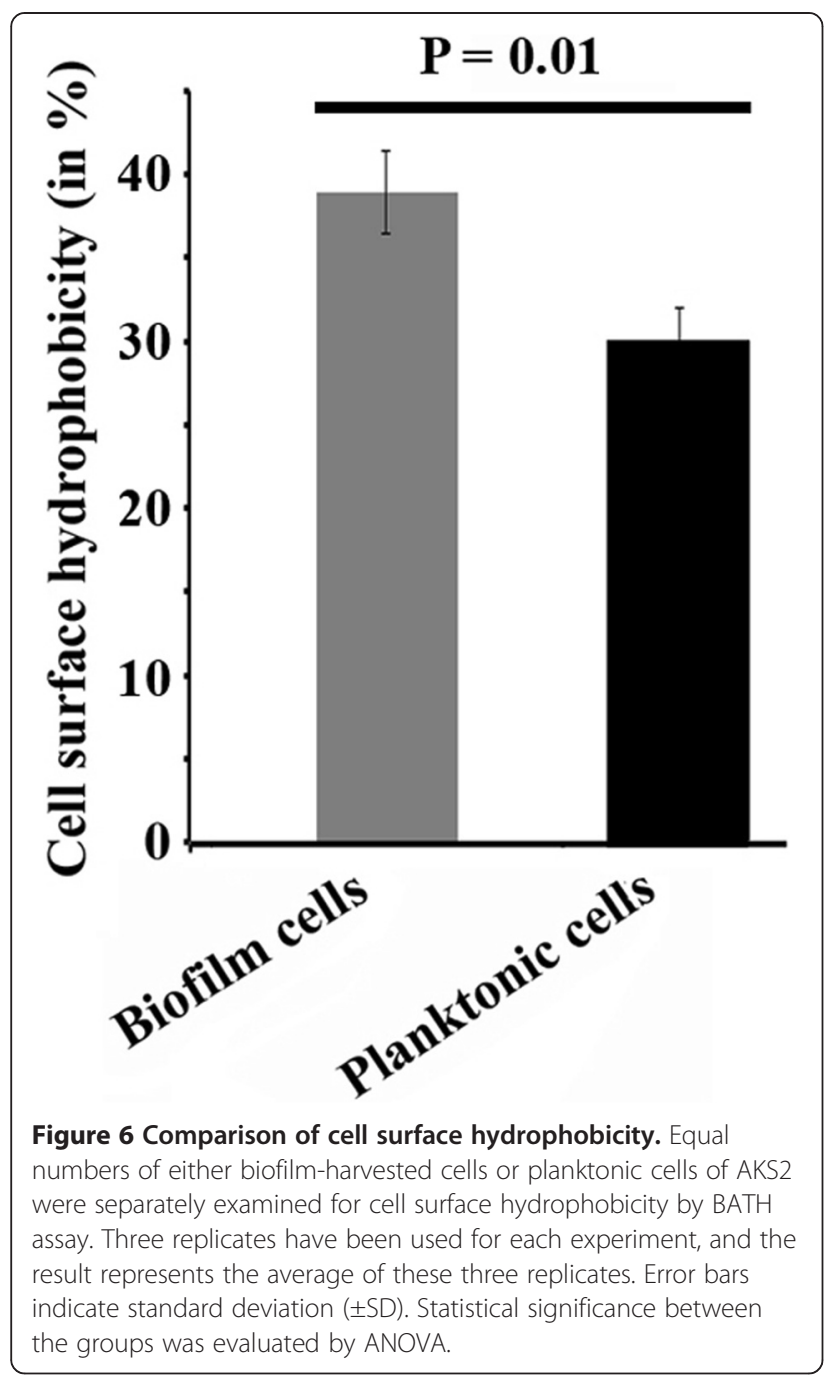

adaptation, we observed that biofilm cells on polyethylene surface exhibited higher metabolic activity, in particular hydrolytic activity, compared to planktonic cells. This increased metabolic activity may help these cells to degrade and utilize the polymer to establish a sustainable population. Biofilm-harvested cells also exhibited increased functional diversity. This indicates that microorganisms are undergoing phenotypic diversifications, which leads to better adaptation. Moreover, biofilm-harvested cells exhibit lower Gini coefficient which is an indicator of increased functional homogeneity. Therefore, majority of the individual AKS2 cells within the population have similar levels of metabolic potential with respect to utilization of a wide range of carbon sources. This enables the individual microorganisms to efficiently grow and establish a stable population in biofilm as the possibility of intra-species competition is greatly reduced. It has been documented that Rhodococcus tolerates extreme conditions by structural adaptations such as the modification of cell membrane and the alteration in cell surface hydrophobicity [11]. We also observed increased cell surface hydrophobicity of biofilm-harvested AKS2 cells compared to their planktonic counterpart. This increased cell surface hydrophobicity enables AKS2 cells to attach to the hydrophobic LDPE surface more efficiently compared to planktonic cells. The emergence of this trait demonstrated the occurrence of structural adaptation in biofilm of AKS2 cells on polyethylene surface. Taken together, the increased metabolic potential, higher level of functional diversity and homogeneity, and the increased surface hydrophobicity resulted in better colonization and higher reproduction potential of biofilmharvested AKS2 cells.

Phenotypic alteration by an organism in an imposed condition has been considered an important strategy for better adaptation [29]. In our previous study, we observed that AKS2 cells in biofilm on LDPE surface
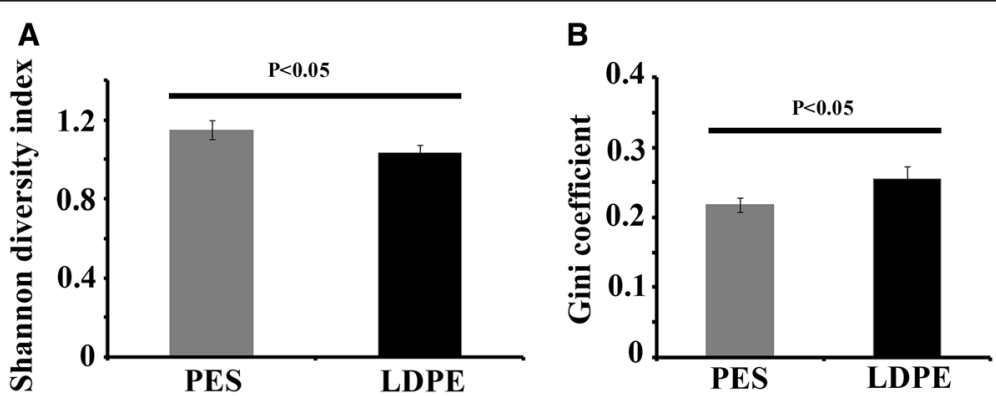

Figure 7 Functional diversity of AKS2 cells on LDPE and PES surface. Equal numbers (approximately $10^{3} \mathrm{CFU}$ ) of biofilm-harvested cells of AKS2, taken from both LDPE and PES surfaces, were aseptically separately added to each well of BiOLOG-ECO plate and incubated for 3 days at $30^{\circ} \mathrm{C}$. Absorbance at $590 \mathrm{~nm}$ of each well was recorded at different time points. Shannon diversity index (A) and Gini coefficient (B) were derived from well colour absorbance at $590 \mathrm{~nm}$ and plotted. Three replicates have been used for each experiment, and the result represents the average of these three replicates. Error bars indicate standard deviation $( \pm S D)$. Statistical significance between the groups was evaluated by ANOVA. 
exhibited a significant alteration in their shape and size compared to the planktonic form [5]. In biofilm, AKS2 cells become more round shaped and smaller in size than planktonic cells [5]. This structural adaptation may provide AKS2 cells a better access to the available nutrients in its surroundings. In the same study, we also observed that AKS2 cells adhering to LDPE secreted exo-polysaccharides for their better attachment and formation of biofilm on the polymer [5]. Again, it suggests the occurrence of physiological adaptations of AKS2 cells on LDPE surface. Collectively, these results demonstrated that AKS2 cells have adapted successfully in biofilm on LDPE surface resulting in a viable population and better degradation of the LDPE polymer.

The stability of an ecosystem depends on the balanced interactions between biotic and abiotic components [23]. These biotic and abiotic components are connected together through nutrient cycles and energy flows [30]. Thus, the abiotic surface of an ecosystem is likely to play a significant role towards adaptation of an organism in a given ecological niche. Towards this, our results showed different levels of functional diversity and evenness of the same organism, AKS2 cells, for two different polymers: LDPE and PES. Thus, the result demonstrates the possible involvement of LDPE towards adaptation of AKS2 cells in biofilm on LDPE surface.

\section{Conclusions}

Towards understanding biofilm-mediated LDPE degradation by AKS2, we verified the adaptation of AKS2 cells in biofilm by examining their viability and fitness. The results showed a viable population of AKS2 cells in biofilm with increased fitness compared to their planktonic counterpart. Further investigation revealed higher metabolic potential, higher functional diversity and homogeneity, and higher level of surface hydrophobicity for the biofilm-harvested AKS2 cells than planktonic cells. All these physiological and structural properties are known to be connected to the adaptability of an organism, and thus, these observations strongly support the view that AKS2 cells have adapted successfully in biofilm and thus developed a viable and stable population which resulted in enhanced polymer degradation. Thus, the current study deciphers the underlying mechanism of LDPE degradation by AKS2 biofilm cells with an enhanced rate.

In conclusion, the current study demonstrates structural and physiological adaptation of AKS2 cells in biofilm on polyethylene surface wherein the nature of the polymer plays an important role. This adaptation leads to enhanced LDPE degradation through biofilm formation.

\section{Additional files}

Additional file 1: Figure S1. Scanning electron micrograph. The micrograph shows the formation of biofilm by AKS2 on LDPE surface.

Additional file 2: Figure S2. Bar diagram. The bar diagram shows the extent of LDPE degradation by AKS2.

\section{Abbreviations}

ANOVA: Analysis of variance; AWCD: Average well colour development; BATH: Bacterial adhesion to hydrocarbon; CFU: Colony-forming unit; C-source: Carbon source; FDA: Fluorescein diacetate; G: Gini coefficient; H: Shannon diversity index; L: Lorenz curve; LDPE: Low-density polyethylene; OD: Optical density; PES: Polyethylene succinate; UPGMA: Unweighted pair group mean averages.

\section{Competing interests}

The authors declare that they have no competing interests.

\section{Authors' contributions}

PT and AKS conceived the idea and designed the experiments. PT performed all the experimental works. PT, AD and AKS interpreted the results and wrote the manuscript. All authors read and approved the final manuscript.

\section{Acknowledgements}

We thank Dr. Srimonti Sarkar for critical reading of the manuscript. This work is supported by a grant in aid from the Department of Biotechnology, Government of West Bengal, India (Sanction no. 555-BT (Estt)/RD-21/11).

Received: 14 January 2015 Accepted: 10 March 2015

Published online: 25 March 2015

\section{References}

1. Roy PK, Titus S, Surekha P, Tulsi E, Deshmukh C, Rajagopal C (2008) Degradation of abiotically aged LDPE films containing pro-oxidant by bacterial consortium. Polym Degrad Stab 93:1917-1922

2. Chatterjee S, Roy B, Roy D, Banerjee R (2010) Enzyme-mediated biodegradation of heat treated commercial polyethylene by Staphylococcal species. Polym Degrad Stab 95:195-200

3. Albertsson AC, Erlandsson B, Hakkarainen M, Karlsson S (1998) Molecular weight changes and polymeric matrix changes correlated with the formation of degradation products in biodegraded polyethylene. J Environ Polym Degrad 6:187-195

4. Volke-Sepulveda T, Saucedo-Castaneda G, Gutierrez-Rojas M, Manzur A, Favela-Torres E (2002) Thermally treated low density polyethylene biodegradation by Penicillium pinophilum and Aspergillus niger. J Appl Polym Sci 83:305-314

5. Tribedi P, Sil AK (2013) Low-density polyethylene degradation by Pseudomonas sp. AKS2 biofilm. Environ Sci Pollut Res Int 20:4146-4153

6. Cvitkovitch DG, Li YH, Ellen RP (2003) Quorum sensing and biofilm formation in streptococcal infections. J Clin Investig 112:1626-1632

7. Kim J, Kim HS, Han S, Lee JY, Oh JE, Chung S, Park HD (2013) Hydrodynamic effects on bacterial biofilm development in a microfluidic environment. Lab Chip 13:1846-1849

8. Dobzhansky T, Hecht MK, Steere WC (1968) On some fundamental concepts of evolutionary biology. In: Evolutionary biology volume 2 (1st edition). Appleton-Century-Crofts, New York, pp 1-34

9. Li YH, Hanna MN, Svensater G, Ellen RP, Cvitkovitch DG (2001) Cell density modulates acid adaptation in Streptococcus mutans: implications for survival in biofilms. J Bacteriol 183:6875-6884

10. Dash HR, Mangwani N, Chakraborty J, Kumari S, Das S (2013) Marine bacteria: potential candidates for enhanced bioremediation. Appl Microbiol Biotechnol 97:561-571

11. de Carvalho CCCR (2012) Adaptation of Rhodococcus erythropolis cells for growth and bioremediation under extreme conditions. Res Microbiol 163:125-136 
12. Tribedi P, Sarkar S, Mukherjee K, Sil AK (2012) Isolation of a novel Pseudomonas sp. from soil that can efficiently degrade polyethylene succinate. Environ Sci Pollut Res Int 19:2115-2124

13. Chrzanowski TH, Crotty RD, Hubbard JG, Welch RP (1984) Applicability of the fluorescein diacetate method of detecting active bacteria in freshwater. Microb Ecol 10(2):179-185

14. Rosenberg M, Perry A, Bayer EA, Gutnick DL, Rosenberg E, Ofek I (1981) Adherence of Acinetobacter calcoaceticus RAG-1 to human epithelial cells and to hexadecane. Infect Immun 33:29-33

15. Choi KH, Dobbs FC (1999) Comparison of two kinds of BiOLOG microplates (GN and ECO) in their ability to distinguish among aquatic microbial communities. J Microbiol Method 36:203-213

16. Garland JL (1997) Analysis and interpretation of community-level physiological profiles in microbial ecology. FEMS Microbiol Ecol 24:289-300

17. Tilman D (2001) Functional Diversity. pp. 109-120. In: Encyclopedia of Biodiversity. Volume 3 (Levin, S.A., ed.). Academic Press, San Diego, 870 pp.

18. Teng Y, Luo Y, Sun M, Liu Z, Li Z, Christie P (2010) Effect of bioaugmentation by Paracoccus sp. strain HPD-2 on the soil microbial community and removal of polycyclic aromatic hydrocarbons from an aged contaminated soil. Bioresour Technol 101:3437-3443

19. Tribedi P, Sil AK (2013) Bioaugmentation of polyethylene succinate-contaminated soil with Pseudomonas sp. AKS2 results in increased microbial activity and better polymer degradation. Environ Sci Pollut Res Int 20:1318-1326

20. Killham K, Staddon WJ (2002) Bioindicators and sensors of soil health and the application of geostatistics. In: Burns RG, Dick R (eds) Enzymes in the environment: activity, ecology and applications. Marcel Dekker, New York, pp 391-405

21. Gilan(Orr) I, Hadar Y, Sivan A (2004) Colonization, biofilm formation and biodegradation of polyethylene by a strain of Rhodococcus ruber. Appl Microbiol Biotechnol 65:97-104

22. Balasubramanian V, Natarajan K, Hemambika B, Ramesh N, Sumathi CS, Kottaimuthu R, Rajash KV (2010) High-density polyethylene (HDPE)-degrading potential bacteria from marine ecosystem of Gulf of Mannar, India. Lett Appl Microbiol 51:205-211

23. Chapin FS, Pamela AM, Harold AM (2002) Principles of terrestrial ecosystem ecology. Springer, New York, ISBN 0-387-95443-0

24. deBeer D, Stoodley P, Roe F, Lewandowski Z (1994) Effects of biofilm structure on oxygen distribution and mass transport. Biotechnol Bioeng 43:1131-1138

25. Stoodley P, Sauer K, Davies DG, Costerton JW (2002) Biofilms as complex differentiated communities. Annu Rev Microbiol 56:187-209

26. Aertsen A, Michiels CW (2004) Stress and how bacteria cope with death and survival. Crit Rev Microbiol 30:263-273

27. Boles BR, Thoendel M, Singh PK (2004) Self-generated diversity produces "insurance effects" in biofilm communities. Proc Natl Acad Sci U S A 101:16630-16635

28. Koh KS, Lam KW, Alhede M, Queck SY, Labbate M, Kjelleberg S, Rice SA (2007) Phenotypic diversification and adaptation of Serratia marcescens MG1 biofilm-derived morphotypes. J Bacteriol 189:119-130

29. Price TD, Qvarnstrom A, Irwin DE (2003) The role of phenotypic plasticity in driving genetic evolution. Proc R Soc Lond B 270:1433-1440

30. Odum EP (1971) Fundamentals of ecology, 3rd edn. Saunders, New York ISBN 0534420664

\section{Submit your manuscript to a SpringerOpen ${ }^{\odot}$ journal and benefit from:}

- Convenient online submission

- Rigorous peer review

- Immediate publication on acceptance

- Open access: articles freely available online

- High visibility within the field

- Retaining the copyright to your article

Submit your next manuscript at $>$ springeropen.com 